\title{
H2S Donor and Bone Metabolism
}

\author{
Yanming Hao ${ }^{1}$, Hongzhen Wang ${ }^{1}$, Lingna Fang ${ }^{2}$, Jinsong Bian ${ }^{3}$, Yan Gao ${ }^{1 *}$ and Chong $\mathrm{Li}^{1 *}$ \\ ${ }^{1}$ Department of Orthopedics, the First Peoples' Hospital of Kunshan, Kunshan, China, ${ }^{2}$ Department of Endocrinology, the First \\ Peoples' Hospital of Kunshan, Kunshan, China, ${ }^{3}$ Department of Pharmacology, Southern University of Science and Technology, \\ Shenzhen, China
}

Hydrogen sulfide $(\mathrm{H} 2 \mathrm{~S})$ has been recognized as the third gasotransmitter, following nitric oxide and carbon monoxide, and it exerts important biological effects in the body. Growing evidence has shown that H2S is involved in many physiological processes in the body. In recent years, much research has been carried out on the role of $\mathrm{H} 2 \mathrm{~S}$ in bone metabolism. Bone metabolic diseases have been linked to abnormal endogenous H2S functions and metabolism. It has been found that H2S plays an important role in the regulation of bone diseases such as osteoporosis and osteoarthritis. Regulation of H2S on bone metabolism has many interacting signaling pathways at the molecular level, which play an important role in bone formation and absorption. H2S releasing agents (donors) have achieved significant effects in the treatment of metabolic bone diseases such as osteoporosis and

OPEN ACCESS

Edited by:

Jinsong Bian,

National University of Singapore,

Singapore

Reviewed by:

Xu Cao,

Beckman Research Institute,

United States

Guangdong Yang,

Laurentian University, Canada

*Correspondence:

Yan Gao

ygao1106@alu.suda.edu.cn

Chong $\mathrm{Li}$

lichong1705@163.com

Specialty section: This article was submitted to Translational Pharmacology, a section of the journal Frontiers in Pharmacology

Received: 31 January 2021 Accepted: 05 July 2021 Published: 22 July 2021

Citation:

Hao Y, Wang H, Fang L, Bian J, Gao Y and Li C (2021) H2S Donor and

Bone Metabolism.

Front. Pharmacol. 12:661601. doi: 10.3389/fphar.2021.661601 osteoarthritis. In addition, $\mathrm{H} 2 \mathrm{~S}$ donors and related drugs have been widely used as research tools in basic biomedical research and may be explored as potential therapeutic agents in the future. Donors are used to study the mechanism and function of $\mathrm{H} 2 \mathrm{~S}$ as they release $\mathrm{H} 2 \mathrm{~S}$ through different mechanisms. Although $\mathrm{H} 2 \mathrm{~S}$ releasers have biological activity, their function can be inconsistent. Additionally, donors have different H2S release capabilities, which could lead to different effects. Side effects may form with the formation of H2S; however, it is unclear whether these side effects affect the biological effects of H2S. Therefore, it is necessary to study H2S donors in detail. In this review, we summarize the current information about $\mathrm{H} 2 \mathrm{~S}$ donors related to bone metabolism diseases and discuss some mechanisms and biological applications.

Keywords: bone metabolism, mechanisms, diseases, hydrogen sulfide, donor

\section{INTRODUCTION}

With the development of aging society, the incidence of bone metabolic diseases is increasing year by year, such as osteoporosis and osteoarthritis, which seriously affects the quality of life of the elderly. Osteoporosis is a systemic bone disease characterized by osteopenia, destruction of bone microstructure and decrease of bone strength, resulting in the increase of bone fragility and fracture. Osteoblasts and osteoclasts play an important role in the process of bone metabolism. At present, the main mechanisms of osteoporosis drugs include inhibiting bone resorption, promoting bone formation, other mechanisms and traditional Chinese medicine, such as bisphosphonates, estrogen and parathyroid hormone (PTH), etc. However, long-term use of bisphosphonates can lead to jaw necrosis and atypical fractures of long bones (Hollick and Reid, 2011). Recombinant thyroid hormone (PTH) has been shown to directly promote bone formation, but at the same time stimulate osteoclast production and increase bone resorption (Wang et al., 2007). The large use of estrogen replacement therapy and its analogues will increase the risk of cardiovascular events or breast cancer in osteoporotic patients (Thorbjarnardottir et al., 2014). 
Osteoarthritis is a kind of joint degenerative disease with joint pain, deformity and dysfunction, which is the most common form of arthritis and the main cause of disability in the elderly. Although a variety of metabolic, genetic, epigenetic and local factors are involved, the complete etiology is still unclear. At present, there is no cure for this kind of pathology, and we are actively looking for alternative treatments to delay or prevent its progress. Therefore, it is of great clinical significance to find a new treatment.

As a newly discovered gaseous signaling molecule, $\mathrm{H} 2 \mathrm{~S}$ plays an important role in the cardiovascular, nervous, metabolic, neuroendocrine, and bone metabolism systems (Wang, 2010). In mammalian tissues, $\mathrm{H} 2 \mathrm{~S}$ is synthesized endogenously by three tissue-specific enzymes: cystathionine $\gamma$-lyase (CSE), cystathionine $\beta$-synthase (CBS), and 3-mercapto-pyruvate sulfurtransferase (3MST) (Singh et al., 2009). The first two enzymes are pyridoxal 5phosphate (PLP)-dependent, using L-cysteine (Cys) and homocysteine (Hcy) as substrates and are catalyzed by CBS and CSE; the third is in the pyridoxal- $5^{\prime}$-phosphate (PLP)-independent 3-mercaptopyruvate sulfurtransferase (MST) pathway, which uses sulfur-containing amino acids as substrates (Gallego et al., 2008). The metabolic pathway of H2S in the body operates through the oxidation of mitochondria to produce sulfate and thiosulfate, the final products of which are ammonium and pyruvate (Olson, 2012). To generate enough Adenosine triphosphate (ATP) from the mitochondrial oxidation of $\mathrm{H} 2 \mathrm{~S}$, sufficient exogenous $\mathrm{H} 2 \mathrm{~S}$ needs to be present. Endogenous hydrogen sulfide increases the production of ATP by promoting S-sulfhydration of the mitochondrial inner membrane protein ATP synthase, and supplementation of exogenous hydrogen sulfide donor NaHS can also promote the production of ATP (Módis et al., 2016). In animals, H2S has been proved to be the electron source of mitochondrial ATP synthesis. At lower concentrations, $\mathrm{H} 2 \mathrm{~S}$ can supply electrons in the electron transport chain, thus increasing the yield of ATP (Bouillaud et al., 2013; Helmy et al., 2014). H2S is the endogenous activator of ATP-dependent potassium channel (KATP channel) (Módis et al., 2013). Excess H2S in the body can be removed by molecules containing metals and sulfides, such as peroxides, catalase, and glutathione oxides (Wang, 2012). It is worth noting that catalytic oxidation of $\mathrm{H} 2 \mathrm{~S}$ is the main mechanism for the regulation of H2S levels in cells (Hildebrandt and Grieshaber, 2008).

The lipid-soluble capability of $\mathrm{H} 2 \mathrm{~S}$ is five times that of its watevr solubility; therefore, $\mathrm{H} 2 \mathrm{~S}$ can pass quickly through the cell membranes without the need for any specific transporter or receptor. H2S promotes a number of cellular signals that regulate neurological, cardiac, and respiratory functions, as well as cell metabolism and survival (Mathai et al., 2009). The level of hydrogen sulfide in mammals still remain controversial, various reports describe $\mathrm{H} 2 \mathrm{~S}$ in the blood of mammals, mainly in the range of micromolar concentrations. In healthy animals and humans, the physiological range of $\mathrm{H} 2 \mathrm{~S}$ in circulation is estimated to be 10-100 $\mu \mathrm{mol}$ (Módis et al., 2013). Recent research have shown that the physiological concentration of $\mathrm{H} 2 \mathrm{~S}$ in the peripheral blood ranges from 30 to $100 \mu \mathrm{mol} / \mathrm{L}$, and the $\mathrm{H} 2 \mathrm{~S}$ level in the mammalian brain is $50-160 \mu \mathrm{mol} / \mathrm{L}$ (Sunzini et al., 2020). At present, there is no direct evidence on the level of endogenous hydrogen sulfide in bone tissue. Only Burguera et al. have reported the biosynthesis of $\mathrm{H} 2 \mathrm{~S}$ in articular cartilage. The concentration of hydrogen sulfide in cartilage of osteoarthritis patients was $0.056(0.016-0.080) \mathrm{nmol} / \mathrm{g}$, and in healthy cartilage was $0.457(0.296,0.593) \mathrm{nmol} / \mathrm{g}$ (Burguera et al., 2020). In addition, Liu et al. found that BMMSCs expressed CBS and CSE, and produced $\mathrm{H} 2 \mathrm{~S}$ at the level of 8-10 $\mu \mathrm{mol}$ in the culture supernatant of BMMSC (Liu et al., 2014).

As a reductant, physiological concentrations of $\mathrm{H} 2 \mathrm{~S}$ exert antioxidant, anti-inflammatory, and anti-apoptotic properties (Kimura et al., 2010). Endogenous H2S in the plasma is negatively associated with aging and oxidative stress levels (Hine et al., 2015). Osteoporosis makes bones fragile and leads to an increased risk of bone fractures. Reactive oxygen species (ROS) are important factors during osteoporosis development (Manolagas, 2010). Studies have found that high levels of lipid peroxidation and low antioxidant enzyme activities are present in postmenopausal osteoporotic women and ovariectomized rats (Muthusami et al., 2005). Osteoblasts and osteoclasts play an important role in bone homeostasis, and osteoblasts are responsible for bone formation and mineralization, and bone resorption, respectively. In osteoporosis patients, the dynamic balance between bone resorption and bone formation is disrupted. ROS accelerate osteoblast apoptosis, inhibit their differentiation, and impair bone formation (Charman, 1989). To understand the biological function of $\mathrm{H} 2 \mathrm{~S}$, it is necessary to carry out continuous research related to $\mathrm{H} 2 \mathrm{~S}$. To study the mechanism and function of $\mathrm{H} 2 \mathrm{~S}$, the $\mathrm{H} 2 \mathrm{~S}$ donor is an important research tool, and the $\mathrm{H} 2 \mathrm{~S}$ releasing ability of each donor type is quite different, which may lead to different results. A large number of H2S donors have been studied because of their therapeutic potential. Here, we summarize several H2S donors and their effects on bone metabolism (Table 1).

\section{H2S DONORS}

\section{Sulfide Salt with Bone Metabolism}

Inorganic sulfurized salts, such as sodium sulfide $\left(\mathrm{Na}_{2} \mathrm{~S}\right)$ and sodium H2S (NaHS), are widely used as H2S equivalents in research, as well as being the earliest donors of H2S studied. By using these sulfurized salts, some biological functions regulated by $\mathrm{H} 2 \mathrm{~S}$ have been determined (Whiteman et al., 2010).

The most common H2S donors are sodium sulfide and sodium hydrosulfide (Jimenez, 2010), which can be used to prepare H2S standard solutions. Sodium sulfide has the advantage of rapidly increasing the concentration of $\mathrm{H} 2 \mathrm{~S}$. In addition, they are "clean" donors, because they do not form by-products after the release of $\mathrm{H} 2 \mathrm{~S}$. NaHS improves cell viability, reduces apoptosis induced by $\mathrm{H}_{2} \mathrm{O}_{2}$, and promotes osteoblast proliferation by enhancing the transcription and activity of alkaline phosphatase in MC3T3-E1 osteoblasts. In addition, NaHS treatment can also stimulate the transcription level of osteocalcin, the main bone matrix protein, and collagen protein expression, the main component of bone tissue. NaHS reverses the decrease in superoxide dismutase activity, active oxygen production, and nicotinamide adenine dinucleotide phosphate (NADPH) oxidase activity in osteoblasts treated with $\mathrm{H}_{2} \mathrm{O}_{2}$. $\mathrm{H} 2 \mathrm{~S}$ protects osteoblasts from oxidative stress-induced cell damage and proliferation and differentiation inhibition through mitogen-activated protein kinase (MAPK) (P38 and ERK1/2)-dependent mechanisms; therefore, H2S may have potential therapeutic value for 
TABLE 1 | Summary of H2S donors.

\begin{tabular}{|c|c|c|c|}
\hline H2S donor & $\begin{array}{l}\text { H2S release } \\
\text { mechanism }\end{array}$ & $\begin{array}{l}\text { Effects } \\
\text { on bone metabolism }\end{array}$ & References \\
\hline Sulfide salt & Rapidly hydrolysis & $\begin{array}{l}\text { Promote osteoblast proliferation } \\
\text { Protect osteoblasts from oxidative stress-induced cell } \\
\text { damage } \\
\text { Stimulate the transcription level of osteocalcin }\end{array}$ & Xu et al. (2011) \\
\hline GYY4137 & $\begin{array}{l}\text { Slowly hydrolysis, } \\
\text { pH-dependent }\end{array}$ & $\begin{array}{l}\text { Prevent DEX-induced bone loss, stimulate the formation } \\
\text { and differentiation of osteoblasts }\end{array}$ & (Xu et al., 2011), (Grassi et al., 2016) \\
\hline Garlic-derived sulfur compounds & Thiol activation & $\begin{array}{l}\text { Protect osteoblasts from ROS formation } \\
\text { Increases the activity of alkaline phosphatase and Ca2+ } \\
\text { ATPase }\end{array}$ & $\begin{array}{l}\text { (Qiu et al., 2015), [Ahmadian et al. (2017)], } \\
\text { (Mukherjee et al., 2006c) }\end{array}$ \\
\hline $\begin{array}{l}\text { Dithiolethiones and their NSAID } \\
\text { hybrids }\end{array}$ & Hydrolysis & Anti-inflammation and inhibit bone defects and bone loss & Herrera et al. (2015) \\
\hline DM-22 & Thiol activation & $\begin{array}{l}\text { Inhibits } \mathrm{H}-\mathrm{OCS} \text { function and promotes osteogenic } \\
\text { differentiation of H-MSCs }\end{array}$ & Martelli et al. (2012) \\
\hline $\begin{array}{l}\text { Sulfide synthesis from traditional } \\
\text { Chinese medicine donor }\end{array}$ & Slowly hydrolysis & Reduce the production of active oxygen & Yan et al. (2017) \\
\hline HNO donors & Hydrolysis & $\begin{array}{l}\text { Produce anti-inflammatory effect } \\
\text { No biological effects on bone metabolism were reported } \\
\text { to date }\end{array}$ & Zhou et al. (2016) \\
\hline Photolabile H2S donors & Light activation & $\begin{array}{l}\text { No biological effects on bone metabolism were reported } \\
\text { to date }\end{array}$ & Fukushima et al. (2014) \\
\hline Self-immolate thiocarbamates & Esterase-triggered & $\begin{array}{l}\text { No biological effects on bone metabolism were reported } \\
\text { to date }\end{array}$ & Steiger et al. (2016) \\
\hline
\end{tabular}

osteoporosis (Xu et al., 2011). In bone loss induced by hyperhomocysteinemia (HHcy), $\mathrm{NaSH}$ epigenetically mitigates bone loss through osteoprotegerin (OPG)/receptor activator of nuclear factor kappa-B ligand (RANKL) regulation (Behera et al., 2018a). Studies report that NaHS decreased the differentiation of human osteoclasts in a dose-dependent manner by up-regulating the expression of nuclear factor erythroid 2-related factor 2 (NRF2) protein (Gambari et al., 2014). The activation of NRF2 may inhibit the differentiation of human osteoclasts by activating the continuous antioxidant response of osteoclast precursors (Gambari et al., 2014). In addition, NaHS can reduce cortical bone loss caused by spinal cord injury, probably by reducing oxidative stress, inhibiting matrix metalloproteinases (MMPs) activity and activating $\mathrm{Wnt} / \beta$-catenin signal transduction (Yang et al., 2017).

NaHS intervention also has an effect on metabolic osteoporosis caused by CBS deficiency. Cysteine $\beta$-amylase heterozygote knockout mice were fed a methionine-rich diet, and the NaHS donor was supplemented for 8 weeks. NaHS treatment was used to normalize the plasma $\mathrm{H} 2 \mathrm{~S}$ and completely prevent trabecular bone loss in CBS+/-mice. The recovery of $\mathrm{H} 2 \mathrm{~S}$ may provide a treatment for metabolic osteoporosis caused by CBS deficiency (Behera et al., 2018b).

However, one of the main problems of such donors is that the release of $\mathrm{H} 2 \mathrm{~S}$ is uncontrollable. Once the sulfate dissolves in the aqueous solution, it releases $\mathrm{H} 2 \mathrm{~S}$ immediately and completely. A large number of rapidly released $\mathrm{H} 2 \mathrm{~S}$ causes a rapid drop in blood pressure and toxic effects in patients and animals (Li et al., 2008).

\section{GYY4137 and Phosphorodithioate-Based Donors with Bone Metabolism}

The morpholin-4-ium 4 methoxyphenyl (morpholino) phosphinodithioate (GYY4137) is a novel water-soluble donor of $\mathrm{H} 2 \mathrm{~S}$, which can release $\mathrm{H} 2 \mathrm{~S}$ slowly, sustaining a controlled release. Some experiments showed that $4.17 \pm 0.5 \mathrm{nmol} / 25 \mathrm{~min}$ of H2S was released from $1 \mathrm{~mm}$ of GYY4137 (Zhao et al., 2015), and the mechanism of release was likely through hydrolysis. In addition, the release is $\mathrm{pH}$-dependent; studies showed signs of maximum release at acidic $\mathrm{pH}$ (3.0) and less release at neutral or basic pH $(7.4,8.5)$ (Martelli et al., 2013). After administering GYY4137 to rats, the concentration of $\mathrm{H} 2 \mathrm{~S}$ in the plasma reached its highest level after $30 \mathrm{~min}$ and lasted for $3 \mathrm{~h}$, indicating that GYY4137 released H2S much more slowly than NaHS. Cytotoxicity tests in rats showed that GYY4137 did not cause significant cell damage for three days at doses up to $100 \mu \mathrm{M}$, while NaHS caused apoptosis in smooth muscle cells at similar doses and times (Baskar et al., 2007). The difference in the experimental results may be explained by the different release rates of the two $\mathrm{H} 2 \mathrm{~S}$ donors. The main advantage of GYY4137 is that H2S can be slowly released.

GYY4137 plays a role in glucocorticoid-induced bone loss. By supplementing GYY4137 with H2S, bone formation can be promoted and bone absorption can be inhibited to prevent dexamethasone (DEX)-induced bone loss. It can also prevent osteoporosis caused by DEX and prevent trabecular and cortical bone loss. The mechanism of action can be achieved by activating the $\mathrm{Wnt} / \beta$-catenin signaling pathway (Ma et al., 2019). GYY4137 activates Wnt signal by increasing the production of Wnt ligands Wnt16, Wnt2b, Wnt6 and Wnt10b in bone marrow, thus increasing the formation of osteoblasts (Grassi et al., 2016). In addition, GYY4137 can enhance osteoblast differentiation by stimulating the transcription level of runt-related transcription factor 2 (Yang et al., 2019a). GYY4137 also protected osteoblasts from apoptosis induced by DEX. These results indicate that GYY4137 is an effective way to prevent and treat osteoporosis 
and osteonecrosis caused by glucocorticoids (GCs) (Ma et al., 2019). In the fracture model, GYY4137 can antagonize the loss of calcium and phosphorus by reducing glucocorticoid secretion and inhibiting the vulcanization of glucocorticoid receptor $a$ (Liao et al., 2021).

GYY4137 can also stimulate the formation and differentiation of osteoblasts through an ERK1/2-dependent antioxidant mechanism and successfully inhibit the oxidative damage of MC3T-E1 induced by hydrogen peroxide (Xu et al., 2011). It has been found that GYY4137 can prevent particle-induced inflammation and osteolysis through SIRT1 pathway during prosthesis loosening (Liu et al., 2020). GYY4137, as a controlled-release donor of $\mathrm{H} 2 \mathrm{~S}$, can simulate the formation of endogenous $\mathrm{H} 2 \mathrm{~S}$ and has more advantages than sodium sulfide as an exogenous donor (Liu et al., 2013). By intraperitoneal injection of GYY4137 as a supplement of endogenous H2S, the physiological level of $\mathrm{H} 2 \mathrm{~S}$ in rat plasma can be improved and maintained to regulate the balance of calcium and phosphorus metabolism. The results showed that GYY4137 was a better intervention drug than alendronate and had a certain therapeutic effect on osteoporosis after oophorectomy (Xu et al., 2018).

\section{Natural Sulfur-Containing Compounds with Bone Metabolism}

Garlic contains rich fat-soluble and water-soluble sulfides and can inherently release $\mathrm{H} 2 \mathrm{~S}$, which has a protective effect against postmenopausal osteoporosis. H2S donors derived from garlic include diallyl sulfide (DAS), diallyl disulfide (DADS), and diallyl trisulfide (DATS) (Amagase, 2006). DAS and s-DADS are the main components of garlic with high antioxidant activity. DADS protects osteoblasts from ROS formation and protects smokers via ROS formation (Qiu et al., 2015). According to previous studies, these sulfur-containing compounds could inhibit the downregulation of the c-Jun $\mathrm{N}$-terminal kinase (JNK) signaling pathway, which may lead to the protective role of S-allyl cysteine in oxidized-LDL formation and inhibition of nuclear factor-kappa B activation by hydrogen peroxide (Malandrino et al., 2012; Dada et al., 2014).

Garlic and its derivatives can be beneficial for osteoporosis to inhibit bone absorption, and it might also have a significant influence on carbonyl groups, advanced oxidation protein products (AOPPs), and ROS. It is likely that garlic prevents osteoporosis by suppressing oxidative stress. Previous research has shown that an increased oxidative stress index was observed in the garlic-treated group, but it was significantly reduced in the carbonyl group and AOPPs after garlic administration (Meisinger et al., 2014; Ahmadian et al., 2017).

The comparison of the anti-osteoporosis potential between garlic and 17- $\beta$-estradiol showed that garlic has the function of preventing and maintaining bone health. Garlic can reduce the excretion of calcium and phosphate and plays an active role in counteracting the increased osteoclast activity and bone absorption due to the lack of ovarian hormones. Garlic has potential antioxidant activity, which may be the main reason for scavenging free radicals produced by a trap. So far as potential is concerned, Garlic's efficacy as a natural therapeutic agent must be valued. In fact, garlic may prove therapeutically better than estrogen when comparing the well-established negative side effects of the use of estrogen as an anti-osteoporotic agent (Mukherjee et al., 2006a). Garlic can significantly increase the calcium transfer of all intestinal segments of bilaterally ovariectomized rats and increases the activity of alkaline phosphatase and $\mathrm{Ca}^{2+}$ ATPase (Mukherjee et al., 2006b).

DATS and DADS, the donors of $\mathrm{H} 2 \mathrm{~S}$, are the two main organic sulfides in garlic oil, which are allicin produced by garlic decomposition, and their health benefits have been widely studied. Garlic tablets not only have a good effect on postmenopausal osteoporosis but also have many beneficial effects on oxidative stress, and can reduce the activity of NADPH oxidase and the expression of vascular cell adhesion molecule 1 (VCAM-1), thereby reducing inflammation. DADS can attenuate inflammatory osteolysis by inhibiting osteoclast formation through NF- $\kappa$ B-NFATc1 signal pathway (Yang et al., 2019b). In patients with arthritis, DADS inhibits the expression of three MMPs induced by IL-1/OncostatinM (OSM) in a dosedependent manner, thus protecting articular cartilage (Williams et al., 2010).

Both chemical management and estrogen therapy have side effects. The advantage of allicin is its inhibitory effect on oxidative stress; other food extracts of onion, ginger, Nigella sativa, and Berberis vulgaris can also be further studied.

\section{1,2-Dithiole-3-Thiones and H2S Hybrid NSAIDs with Bone Metabolism}

1,2-disulfide-3-thioketone (DTT) is recognized as the core of H2S release; it releases $\mathrm{H} 2 \mathrm{~S}$ in aqueous solution, and hydrolysis may be the mechanism of $\mathrm{H} 2 \mathrm{~S}$ production by DTT (Hagle and McKay, 1996). Elemental sulfur or phosphorus pentasulfide is used for the dehydrogenation and sulfurization of allyl methyl to obtain the desired product. In another method, disulfide acids are obtained from the reaction between ketones and carbon disulfide $\left(\mathrm{CS}_{2}\right)$, react with hexamethyl disulfide (HMDT, sulfur resource), and $\mathrm{N}$-chlorosuccinimide (NCS, an oxidant) is replaced by DTTs. In addition, $\beta$-keto esters react with the Lawesson reagent to form DTTs (Kianmehr et al., 2010).

The mixed NSAIDs (HS NSAIDs) produced by DTT combined with NSAIDs significantly reduced gastrointestinal damage compared to maternal NSAIDs (Sparatore et al., 2011; Chan and Wallace, 2013; Gargallo and Lanas, 2013). HS NSAIDs are usually synthesized by coupling NSAIDs with 5-(4hydroxyphenyl)-3h-1,2-disulfide-3-thioone (ADT-OH) in the presence of dicyclohexyl carbodiimide (DCC) and 4dimethylaminopyridine (DMAP). When heated to $120^{\circ} \mathrm{C}$ in dimethyl sulfoxide (DMSO) phosphate buffer mixture, DTT releases $\mathrm{H} 2 \mathrm{~S}$, which indicates that DTT can be regarded as a hydrolytic H2S donor (Qandil, 2012), and is often used to prepare mixed drugs of $\mathrm{H} 2 \mathrm{~S}$ donors. Some of the main candidate drugs have been reported in the fields of inflammation, the cardiovascular system, urinary system, and neurodegenerative diseases. These mixtures show significant improvement in 
activity and side effects, suggesting that $\mathrm{H} 2 \mathrm{~S}$ has useful pharmacological effects (Sparatore et al., 2011); rats receiving the same amount of acs14 did not show gastric injury, and the concentration of $\mathrm{H} 2 \mathrm{~S}$ in plasma increased three times.

ATB-346, a derivative product of $\mathrm{H} 2 \mathrm{~S}$ releasing naproxen, can significantly inhibit bone defects and bone loss caused by ligation. The H2S releasing part of the ATB-346 compound not only does not damage the effect of naproxen on periodontitis, but also improves bone quality, which provides a potential new adjuvant therapy for the treatment of periodontal diseases (Herrera et al., 2015).

Although HS-NSAIDs have been shown to play a role in $\mathrm{H} 2 \mathrm{~S}$ release in many tissues and organs, the mechanism of release of $\mathrm{H} 2 \mathrm{~S}$ in vivo needs to be further studied. Additionally, whether the biological effect of HS-NSAIDs is related to H2S or sulfas needs to be further studied.

\section{Mercaptan-Activated H2S Donor with Bone Metabolism}

Mercaptan-activated H2S donors were first reported in 2011, and are unstable and easily broken to form a hydrosol anion, which is the main form of $\mathrm{H} 2 \mathrm{~S}$ under physiological conditions. The acyl group was used to protect the $-\mathrm{SH}$ residue of N-SH. The resulting compound, $\mathrm{N}$-(benzoylthio) benzamide, was stable in water only in the presence of thiols (cysteine or glutathione). These donors were evaluated in plasma, and similar $\mathrm{H} 2 \mathrm{~S}$ release products were observed (Zhao et al., 2011).

DM-22, a new H2S hybrid n-bps synthesis, can induce the osteogenic differentiation of mesenchymal stromal cells human mesenchymal stem cells (H-MSCs) and maintain anti-osteoclast activity in vitro (Martelli et al., 2012). DM-22 is a slowly released $\mathrm{H} 2 \mathrm{~S}$ donor derived from a combination of alendronate (AL) and aryl isothiocyanates that release a portion of $\mathrm{H} 2 \mathrm{~S}$. It is cultured in an aqueous solution ( $\mathrm{pH} 7.4$ and $20^{\circ} \mathrm{C}$ analysis buffer) for $1 \mathrm{~mm}$, resulting in the production of a time-dependent increase in $\mathrm{H} 2 \mathrm{~S}$ concentration in the presence of $4 \mathrm{~mm} \mathrm{~L}$-cysteine. $\mathrm{H} 2 \mathrm{~S}$ production rises to a stable state after $10 \mathrm{~min}$ and reaches a maximum $\mathrm{H} 2 \mathrm{~S}$ concentration of $42.0 \pm 2.1 \mathrm{M}$ after $20 \mathrm{~min}$. Conversely, in the absence of 1-cysteine, incubation of DM-22 $(1 \mathrm{~mm})$ resulted in a significant reduction in $\mathrm{H} 2 \mathrm{~S}$ levels, which is dependent on the $\mathrm{H} 2 \mathrm{~S}$ release mechanism of organic mercaptan (Rapposelli et al., 2017). The effects of DM-22 and AL on the osteogenic differentiation of human osteoclasts (H-OCS) and H-MSCS were detected at a concentration of $1-33 \mathrm{~nm}$ in vitro. Current measurements show that DM-22 releases $\mathrm{H} 2 \mathrm{~S}$ at a slow and mercaptan-dependent rate. DM-22 significantly inhibited $\mathrm{H}$-OCS differentiation and function, maintaining residual $\mathrm{H}-\mathrm{OCS}$ activity even at high doses of $33 \mu \mathrm{m}$. Furthermore, DM-22 inhibited the differentiation and function of H-OCSs without causing cytotoxicity. DM-22 showed no cytotoxicity over the whole concentration range. The release of H2S by DM-22 has persistent kinetics, which conforms to the characteristics of the isothiocyanate-type H2S-donor (Martelli et al., 2013; Citi et al., 2014). In the design of this hybrid drug, this pharmacological property could be expected to improve the overall pharmacological characteristics and attenuate the possible cytotoxicity of the BPS molecular portion.
DM-22 is a novel cytokine that inhibits H-OCS function and promotes osteogenic differentiation of H-MSCs without cytotoxicity. It is an ideal candidate for a new family of bone anabolic drugs, which would provide new therapeutic opportunities in the field of bone loss.

\section{Sulfide Synthesis from Traditional Chinese Medicine Donor with Bone Metabolism}

SDSS is a representative $\mathrm{H} 2 \mathrm{~S}$ donor synthesized by traditional Chinese medicine; it is a donor synthesized by Danshensu (DSS) and $\mathrm{H} 2 \mathrm{~S}$, both of which have a significant antioxidant effects in different systems. ROS accelerate the apoptosis of osteoblasts, inhibit their differentiation and damage bones (She et al., 2014; Li et al., 2015). Danshensu [ $\beta$-(3,4-dihydroxyphenyl) lactate] is an important water-soluble compound extracted from Salvia miltiorrhiza. It is widely used in the treatment of various microcirculatory disorder-related diseases in China ( $\mathrm{Yu}$ et al., 2012). DSS can reduce inflammation and inhibit ROS formation (Jiang et al., 2015). In addition to these effects, DSS has a beneficial effect on bone formation. For example, previous studies have shown that DSS not only promotes osteoblast differentiation and bone matrix formation but also regulates osteoblast differentiation (Naghibi et al., 2014).

SDSS is also a slow-release donor of H2S. In rats, H2S is released from the plasma approximately $6 \mathrm{~min}$ after intravenous administration. It reaches its maximum value $15 \mathrm{~min}$ after administration and slowly decreases with time. SDSS can release $\mathrm{H} 2 \mathrm{~S}$ in MC3T3-E1 osteoblasts and restore the ability of $\mathrm{H}_{2} \mathrm{O}_{2}$ to inhibit the differentiation of osteoblasts, which is due to the stimulation of bone sialoprotein, runt-related transcription factor-2, collagen expression, alkaline phosphatase activity, and bone nodule formation. The study showed that SDSS could reduce the production of active oxygen induced by $\mathrm{H}_{2} \mathrm{O}_{2}$, play a protective role, and reverse the decrease in superoxide dismutase activity, decrease in glutathione content, and increase in ROS production in $\mathrm{H}_{2} \mathrm{O}_{2}$ treated cells. In addition, SDSS significantly reduced the activation of $\mathrm{p} 38$, ERK1/2, and JNK MAPKs induced by $\mathrm{H}_{2} \mathrm{O}_{2}$, and SDSS also stimulates the phosphatidylinositol 3 kinase/AKT signaling pathway, blocking the pathway and reducing the cell-protective effect of SDSS. Importantly, SDSS had no significant toxic effect on the apoptosis of MC3T3-E1 cells induced by $\mathrm{H}_{2} \mathrm{O}_{2}$ (Yan et al., 2017).

\section{OTHER H2S DONORS}

Besides the above-mentioned H2S donors, some other donors have been studied in other systems and diseases. However, these studies have not referred to bone metabolism, which provides us with research space and direction for further research.

\section{HNO Donors}

$\mathrm{H} 2 \mathrm{~S}$ and NO have many similar functions, and it is speculated that a new hybrid with both $\mathrm{H} 2 \mathrm{~S}$ and NO donors will be more effective than either alone. Therefore, some hybrid compounds (such as NOSH-1) that release NO-H2S were prepared and tested 
(Kashfi, 2014). Bian et al. studied the biological function of HNO in microglia with Danggui salt, a donor of HNO (Zhou et al., 2016). The study of HNO and bone metabolism of H2S donors has not yet been carried out.

\section{Photolabile H2S Donors}

The $\mathrm{H} 2 \mathrm{~S}$ released from the light-controlled $\mathrm{H} 2 \mathrm{~S}$ donor was directly proportional to the light intensity and duration. It is difficult to control the release rate of $\mathrm{H} 2 \mathrm{~S}$ from these donors because the formation of $\mathrm{H} 2 \mathrm{~S}$ depends on the hydrolysis of Gemini mercaptan in water. Some researchers want to develop a suitable light movable protecting group on $\mathrm{H} 2 \mathrm{~S}$, which will provide an $\mathrm{H} 2 \mathrm{~S}$ donor that can be directly controlled by light; in other words, two protecting groups release $\mathrm{H} 2 \mathrm{~S}$ after photolysis, which releases $\mathrm{H} 2 \mathrm{~S}$ without producing any highly active substance and makes $\mathrm{H} 2 \mathrm{~S}$ completely dependent on the release of light (Fukushima et al., 2014). However, the biological application or pharmacological properties of these lightinduced donors have not been reported.

\section{Self-Immolate Thiocarbamates}

Self-immobilized carbamates release amines with payloads and extrude carbon dioxide as a by-product. The formation of thiocarbamates by replacing carbonyl oxygen with sulfur atoms leads to the release of carbonyl sulfur (COS). In the biological environment, $\mathrm{COS}$ is rapidly hydrolyzed by carbonic anhydrase (CA) to $\mathrm{H} 2 \mathrm{~S}$ and $\mathrm{CO}_{2}$. Carbonic anhydrase is a ubiquitous enzyme found in plants and mammalian cells. When COS is added to a deoxygenated aqueous solution containing cafrombovine red cells, H2S is produced rapidly by using the H2S response electrode (Steiger et al., 2016). However, none of these studies have focused on its biological and pharmaceutical effects.

\section{ENDOGENOUS H2S}

Endogenous $\mathrm{H} 2 \mathrm{~S}$ is formed from cysteine and homocysteine by CBS, CSE, and 3-mercaptopyruvate in mammalian cells. Although CBS is predominant in the hippocampus and cerebellum, endogenous $\mathrm{H} 2 \mathrm{~S}$ protects against $\mathrm{H}_{2} \mathrm{O}_{2}$-induced cytotoxicity in MC3T3-E1 osteoblastic cells (Burguera et al., 2020). The experiment demonstrated that bone marrow mesenchymal stem cells (BMMSCs) produce hydrogen sulfide to regulate their self-renewal and osteogenic differentiation, while deficiency of hydrogen sulfide results in defective differentiation of BMMSCs. H2S deficiency leads to an abnormal influx of

\section{REFERENCES}

Ahmadian, F., Mozaffari-Khosravi, H., Azaraein, M. H., Faraji, R., and Zavar-Reza, J. (2017). The Effect of Consumption of Garlic Tablet on Proteins Oxidation Biomarkers in Postmenopausal Osteoporotic Women: A Randomized Clinical Trial. Electron. Physician 9 (11), 5670-5675. doi:10.19082/5670

Amagase, H. (2006). Clarifying the Real Bioactive Constituents of Garlic. J. Nutr. 136 (3 Suppl. 1), 716S-725S. doi:10.1093/jn/136.3.716s intracellular $\mathrm{Ca}^{2+}$ because it reduces the sulfhydrylation of cysteine residues on multiple $\mathrm{Ca}^{2+}$ TRP channels. This reduced $\mathrm{Ca}^{2+}$ flux downregulates PKC/ERK-mediated Wnt/ $\beta$-catenin signaling, which controls the osteogenic differentiation of BMMSCs. Mice deficient in H2S exhibit an osteoporotic phenotype that can be restored by releasing small molecules of H2S (Liu et al., 2014). These results suggest that H2S can regulate BMMSCs, which provides a possible treatment for osteoporosis and other diseases caused by H2S deficiency.

\section{CONCLUSION}

$\mathrm{H} 2 \mathrm{~S}$ is a gaseous neurotransmitter that plays an important role in bone metabolism. Research on $\mathrm{H} 2 \mathrm{~S}$ and bone metabolism involves osteoblasts, osteoclasts, ovariectomized and hormone-induced osteoporosis models, bone metabolism models after spinal cord injury, H2S key enzyme gene knockout models, epigenetic animal studies, and even human clinical studies select different donors. The properties of donors should be carefully considered, including release rate, by-products, and cross-reactions with other biomolecules. Likewise, other technicalities must be considered, such as whether the release of H2S can be well controlled, the release mechanism of H2S, the by-products after $\mathrm{H} 2 \mathrm{~S}$ release, and the biological activity of these byproducts as well as possible interference effects. Thus, we should be cautious and control the experiment carefully if using $\mathrm{H} 2 \mathrm{~S}$ donors, However, the development of $\mathrm{H} 2 \mathrm{~S}$ donors is crucial for understanding the biological function of $\mathrm{H} 2 \mathrm{~S}$. We look forward to seeing more interesting research in this area.

\section{AUTHOR CONTRIBUTIONS}

YH designed this work and contributed to manuscript writing. LF contributed to literature searches and did the language polishing. HW and JB are responsible for correction. YG and CL are responsible for proofreading, critically revising and supervised the project. YG and CL are the co-corresponding authors of this manuscript. All authors approved the manuscript.

\section{FUNDING}

This study was supported by grants from the Suzhou Science and Technology Plan Project (grant nos. 230301 and SYS2020061).

Baskar, R., Li, L., and Moore, P. K. (2007). Hydrogen Sulfide-induces DNA Damage and Changes in Apoptotic Gene Expression in Human Lung Fibroblast Cells. FASEB j. 21 (1), 247-255. doi:10.1096/fj.06-6255com

Behera, J., Kelly, K. E., Voor, M. J., Metreveli, N., Tyagi, S. C., and Tyagi, N. (2018). Hydrogen Sulfide Promotes Bone Homeostasis by Balancing Inflammatory Cytokine Signaling in CBS-Deficient Mice through an Epigenetic Mechanism. Sci. Rep. 8 (1), 15226. doi:10.1038/s41598-018-33149-9

Behera, J., George, A. K., Voor, M. J., Tyagi, S. C., and Tyagi, N. (2018). Hydrogen Sulfide Epigenetically Mitigates Bone Loss through OPG/RANKL Regulation 
during Hyperhomocysteinemia in Mice. Bone 114, 90-108. doi:10.1016/ j.bone.2018.06.009

Bouillaud, F., Ransy, C., Andriamihaja, M., and Blachier, F. (2013). PL11 Sulfide and Mitochondrial Bioenergetics. Nitric Oxide 31, S15-S16. doi:10.1016/ j.niox.2013.06.021

Burguera, E. F., Vela-Anero, Á., Gato-Calvo, L., Vaamonde-García, C., MeijideFaílde, R., and Blanco, F. J. (2020). Hydrogen Sulfide Biosynthesis Is Impaired in the Osteoarthritic Joint. Int. J. Biometeorol. 64 (6), 997-1010. doi:10.1007/ s00484-019-01823-w

Chan, M. V., and Wallace, J. L. (2013). Hydrogen Sulfide-Based Therapeutics and Gastrointestinal Diseases: Translating Physiology to Treatments. Am. J. Physiology-Gastrointestinal Liver Physiol. 305 (7), G467-G473. doi:10.1152/ajpgi.00169.2013

Charman, W. N. (1989). Symptoms in central Serous Retinopathy. Ophthalmic Physiol. Opt. 9 (3), 340-342. doi:10.1111/j.1475-1313.1989.tb00924.x

Citi, V., Martelli, A., Testai, L., Marino, A., Breschi, M., and Calderone, V. (2014). Hydrogen Sulfide Releasing Capacity of Natural Isothiocyanates: Is it a Reliable Explanation for the Multiple Biological Effects of Brassicaceae? Planta Med. 80 (08/09), 610-613. doi:10.1055/s-0034-1368591

Dada, O. A., Uche, E., Akinbami, A., Odesanya, M., John-Olabode, S., Adediran, A., et al. (2014). The Relationship between Red Blood Cell Distribution Width and Blood Pressure in Patients with Type 2 Diabetes Mellitus in Lagos, Nigeria. J. Blood Med. 5, 185-189. doi:10.2147/JBM.S67989

Fukushima, N., Ieda, N., Sasakura, K., Nagano, T., Hanaoka, K., Suzuki, T., et al. (2014). Synthesis of a Photocontrollable Hydrogen Sulfide Donor Using Ketoprofenate Photocages. Chem. Commun. 50 (5), 587-589. doi:10.1039/ c3cc47421f

Gallego, d., clavé, p., Donovan, j., Rahmati, r., Grundy, d., jiménez, m., et al. (2008). The Gaseous Mediator, Hydrogen Sulphide, Inhibitsin Vitromotor Patterns in the Human, Rat and Mouse colon and Jejunum. Neurogastroenterol Motil. 20 (12), 1306-1316. doi:10.1111/j.1365-2982.2008.01201.x

Gambari, L., Lisignoli, G., Cattini, L., Manferdini, C., Facchini, A., and Grassi, F. (2014). Sodium Hydrosulfide Inhibits the Differentiation of Osteoclast Progenitor Cells via NRF2-dependent Mechanism. Pharmacol. Res. 87, 99-112. doi:10.1016/j.phrs.2014.06.014

Gargallo, C. J., and Lanas, A. (2013). Is NSAIDs-Related Gastrointestinal Damage Preventable? J. Dig. Dis. 14 (2), 55-61. doi:10.1111/1751-2980.12019

Grassi, F., Tyagi, A. M., Calvert, J. W., Gambari, L., Walker, L. D., Yu, M., et al. (2016). Hydrogen Sulfide Is a Novel Regulator of Bone Formation Implicated in the Bone Loss Induced by Estrogen Deficiency. J. Bone Miner Res. 31 (5), 949-963. doi:10.1002/jbmr.2757

Hagle, M. P., and McKay, J. P. (1996). "Turbine Cooling Flow Modulation Apparatus," in Google Patents. doi:10.4135/9781412983495

Helmy, N., Prip-Buus, C., Vons, C., Lenoir, V., Abou-Hamdan, A., GuedouariBounihi, H., et al. (2014). Oxidation of Hydrogen Sulfide by Human Liver Mitochondria. Nitric Oxide 41, 105-112. doi:10.1016/j.niox.2014.05.011

Herrera, B., Coimbra, L., da Silva, A., Teixeira, S., Costa, S., Wallace, J., et al. (2015). The H2S-Releasing Naproxen Derivative, ATB-346, Inhibits Alveolar Bone Loss and Inflammation in Rats with Ligature-Induced Periodontitis. Med. Gas Res. 5, 4. doi:10.1186/s13618-015-0025-3

Hildebrandt, T. M., and Grieshaber, M. K. (2008). Three Enzymatic Activities Catalyze the Oxidation of Sulfide to Thiosulfate in Mammalian and Invertebrate Mitochondria. FEBS J. 275 (13), 3352-3361. doi:10.1111/j.17424658.2008.06482.x

Hine, C., Harputlugil, E., Zhang, Y., Ruckenstuhl, C., Lee, B. C., Brace, L., et al. (2015). Endogenous Hydrogen Sulfide Production Is Essential for Dietary Restriction Benefits. Cell 160 (1-2), 132-144. doi:10.1016/j.cell.2014.11.048

Hollick, R. J., and Reid, D. M. (2011). Role of Bisphosphonates in the Management of Postmenopausal Osteoporosis: an Update on Recent Safety Anxieties. Menopause Int. 17 (2), 66-72. doi:10.1258/mi.2011.011014

Jiang, X., Lv, B., Li, P., Ma, X., Wang, T., Zhou, Q., et al. (2015). Bioactivityintegrated UPLC/Q-TOF-MS of Danhong Injection to Identify NF-Kb Inhibitors and Anti-inflammatory Targets Based on Endothelial Cell Culture and Network Pharmacology. J. Ethnopharmacology 174, 270-276. doi:10.1016/ j.jep.2015.08.026

Jimenez, M. (2010). Hydrogen Sulfide as a Signaling Molecule in the Enteric Nervous System. Neurogastroenterol Motil. 22 (11), 1149-1153. doi:10.1111/ j.1365-2982.2010.01600.x
Kashfi, K. (2014). Anti-cancer Activity of New Designer Hydrogen SulfideDonating Hybrids. Antioxid. Redox Signaling 20 (5), 831-846. doi:10.1089/ ars.2013.5308

Kianmehr, E., Mirza Agha, M., and Estiri, H. (2010). Reaction of Ammonium Ylides with Alkyl Thiocyanates in Aqueous and Non-aqueous media. Monatsh Chem. 141 (4), 409-411. doi:10.1007/s00706-010-0274-8

Kimura, Y., Goto, Y.-I., and Kimura, H. (2010). Hydrogen Sulfide Increases Glutathione Production and Suppresses Oxidative Stress in Mitochondria. Antioxid. Redox Signaling 12 (1), 1-13. doi:10.1089/ars.2008.2282

Li, J., He, W., Liao, B., and Yang, J. (2015). FFA-ROS-P53-mediated Mitochondrial Apoptosis Contributes to Reduction of Osteoblastogenesis and Bone Mass in Type 2 Diabetes Mellitus. Sci. Rep. 5, 12724. doi:10.1038/srep12724

Li, L., Whiteman, M., Guan, Y. Y., Neo, K. L., Cheng, Y., Lee, S. W., et al. (2008). Characterization of a Novel, Water-Soluble Hydrogen Sulfide-Releasing Molecule (GYY4137). Circulation 117 (18), 2351-2360. doi:10.1161/ circulationaha.107.753467

Liao, F., Zhu, Z., Xiao, C., Yuan, J., Geng, B., and Hu, J. (2021). Hydrogen Sulfide Inhibits Calcium and Phosphorus Loss after Fracture by Negatively Regulating Glucocorticoid/glucocorticoid Receptor a. Life Sci. 274, 119363. doi:10.1016/ j.lfs. 2021.119363

Liu, L., Zhou, M., Zhu, R., Zhou, J., Ni, L., Wang, Z., et al. (2020). Hydrogen Sulfide Protects against Particle-induced Inflammatory Response and Osteolysis via SIRT1 Pathway in Prosthesis Loosening. FASEB j. 34 (3), 3743-3754. doi:10.1096/fj.201900393rr

Liu, Y., Yang, R., Liu, X., Zhou, Y., Qu, C., Kikuiri, T., et al. (2014). Hydrogen Sulfide Maintains Mesenchymal Stem Cell Function and Bone Homeostasis via Regulation of Ca2+ Channel Sulfhydration. Cell Stem Cell 15 (1), 66-78. doi:10.1016/j.stem.2014.03.005

Liu, Z., Han, Y., Li, L., Lu, H., Meng, G., Li, X., et al. (2013). The Hydrogen Sulfide Donor, GYY4137, Exhibits Anti-atherosclerotic Activity in High Fat Fed Apolipoprotein E-/-mice. Br. J. Pharmacol. 169 (8), 1795-1809. doi:10.1111/bph.12246

Ma, J., Shi, C., Liu, Z., Han, B., Guo, L., Zhu, L., et al. (2019). Hydrogen Sulfide Is a Novel Regulator Implicated in Glucocorticoids-Inhibited Bone Formation. Aging 11 (18), 7537-7552. doi:10.18632/aging.102269

Malandrino, N., Wu, W. C., Taveira, T. H., Whitlatch, H. B., and Smith, R. J. (2012). Association between Red Blood Cell Distribution Width and Macrovascular and Microvascular Complications in Diabetes. Diabetologia 55 (1), 226-235. doi:10.1007/s00125-011-2331-1

Manolagas, S. C. (2010). From Estrogen-Centric to Aging and Oxidative Stress: a Revised Perspective of the Pathogenesis of Osteoporosis. Endocr. Rev. 31 (3), 266-300. doi:10.1210/er.2009-0024

Martelli, A., Testai, L., Breschi, M. C., Blandizzi, C., Virdis, A., Taddei, S., et al. (2012). Hydrogen Sulphide: Novel Opportunity for Drug Discovery. Med. Res. Rev. 32 (6), 1093-1130. doi:10.1002/med.20234

Martelli, A., Testai, L., Citi, V., Marino, A., Pugliesi, I., Barresi, E., et al. (2013). Arylthioamides as H2S Donors: L-Cysteine-Activated Releasing Properties and Vascular Effects In Vitro and In Vivo. ACS Med. Chem. Lett. 4 (10), 904-908. doi:10.1021/ml400239a

Mathai, J. C., Missner, A., Kugler, P., Saparov, S. M., Zeidel, M. L., Lee, J. K., et al. (2009). No Facilitator Required for Membrane Transport of Hydrogen Sulfide. Proc. Natl. Acad. Sci. 106 (39), 16633-16638. doi:10.1073/ pnas.0902952106

Meisinger, C., Rückert, I., Stöckl, D., Thorand, B., Peters, A., Kowall, B., et al. Hematological Parameters and Prediabetes and Diabetes in Adults from the General Population: a Cross-Sectional Study. Journal of Diabetes \& Metabolism. 2014, 5(2):335. 10.4172/2155-6156.1000335 .

Módis, K., Ju, Y., Ahmad, A., Untereiner, A. A., Altaany, Z., Wu, L., et al. (2016). SSulfhydration of ATP Synthase by Hydrogen Sulfide Stimulates Mitochondrial Bioenergetics. Pharmacol. Res. 113 (Pt A), 116-124. doi:10.1016/ j.phrs.2016.08.023

Módis, K., Wolanska, K., and Vozdek, R. (2013). Hydrogen Sulfide in Cell Signaling, Signal Transduction, Cellular Bioenergetics and Physiology in C. elegans. gpb 32 (1), 1-22. doi:10.4149/gpb_2013001

Mukherjee, M., Das, A. S., Das, D., Mukherjee, S., Mitra, S., and Mitra, C. (2006). Effects of Garlic Oil on Postmenopausal Osteoporosis Using Ovariectomized Rats: Comparison with the Effects of Lovastatin and 17 3 -Estradiol. Phytother. Res. 20 (1), 21-27. doi:10.1002/ptr.1795 
Mukherjee, M., Das, A. S., Das, D., Mukherjee, S., Mitra, S., and Mitra, C. (2006). Role of Oil Extract of Garlic (Allium Sativum Linn.) on Intestinal Transference of Calcium and its Possible Correlation with Preservation of Skeletal Health in an Ovariectomized Rat Model of Osteoporosis. Phytother. Res. 20 (5), 408-415. doi:10.1002/ptr.1888

Mukherjee, M., Das, A. S., Das, D., Mukherjee, S., Mitra, S., and Mitra, C. (2006). Role of Oil Extract of Garlic (Allium Sativum Linn.) on Intestinal Transference of Calcium and its Possible Correlation with Preservation of Skeletal Health in an Ovariectomized Rat Model of Osteoporosis. Phytother. Res. 20 (5), 408-415. doi:10.1002/ptr.1888

Muthusami, S., Ramachandran, I., Muthusamy, B., Vasudevan, G., Prabhu, V., Subramaniam, V., et al. (2005). Ovariectomy Induces Oxidative Stress and Impairs Bone Antioxidant System in Adult Rats. Clin. Chim. Acta 360 (1-2), 81-86. doi:10.1016/j.cccn.2005.04.014

Naghibi, F., Khalaj, A., Mosaddegh, M., Malekmohamadi, M., and HamzelooMoghadam, M. (2014). Cytotoxic Activity Evaluation of Some Medicinal Plants, Selected from Iranian Traditional Medicine Pharmacopoeia to Treat Cancer and Related Disorders. J. Ethnopharmacology 155 (1), 230-239. doi:10.1016/j.jep.2014.05.025

Olson, K. R. (2012). Mitochondrial Adaptations to Utilize Hydrogen Sulfide for Energy and Signaling. J. Comp. Physiol. B 182 (7), 881-897. doi:10.1007/s00360012-0654-y

Qandil, A. (2012). Prodrugs of Nonsteroidal Anti-inflammatory Drugs (NSAIDs), More Than Meets the Eye: a Critical Review. Ijms 13 (12), 17244-17274. doi:10.3390/ijms131217244

Qiu, M., Shen, W., Song, X., Ju, L., Tong, W., Wang, H., et al. (2015). Effects of Prediabetes Mellitus Alone or Plus Hypertension on Subsequent Occurrence of Cardiovascular Disease and Diabetes Mellitus. Hypertension 65 (3), 525-530. doi:10.1161/hypertensionaha.114.04632

Rapposelli, S., Gambari, L., Digiacomo, M., Citi, V., Lisignoli, G., Manferdini, C., et al. (2017). A Novel H2S-Releasing Amino-Bisphosphonate Which Combines Bone Anti-catabolic and Anabolic Functions. Sci. Rep. 7 (1), 11940. doi:10.1038/ s41598-017-11608-Z

She, C., Zhu, L. Q., Zhen, Y. F., Wang, X. D., and Dong, Q. R. (2014). Activation of AMPK Protects against Hydrogen Peroxide-Induced Osteoblast Apoptosis through Autophagy Induction and NADPH Maintenance: New Implications for Osteonecrosis Treatment? Cell Signal. 26 (1), 1-8. doi:10.1016/ j.cellsig.2013.08.046

Singh, S., Padovani, D., Leslie, R. A., Chiku, T., and Banerjee, R. (2009). Relative Contributions of Cystathionine $\beta$-Synthase and $\gamma$-Cystathionase to $\mathrm{H} 2 \mathrm{~S}$ Biogenesis via Alternative Trans-sulfuration Reactions. J. Biol. Chem. 284 (33), 22457-22466. doi:10.1074/jbc.m109.010868

Sparatore, A., Santus, G., Giustarini, D., Rossi, R., and Del Soldato, P. (2011). Therapeutic Potential of New Hydrogen Sulfide-Releasing Hybrids. Expert Rev. Clin. Pharmacol. 4 (1), 109-121. doi:10.1586/ecp.10.122

Steiger, A. K., Pardue, S., Kevil, C. G., and Pluth, M. D. (2016). Self-Immolative Thiocarbamates Provide Access to Triggered H2S Donors and Analyte Replacement Fluorescent Probes. J. Am. Chem. Soc. 138 (23), 7256-7259. doi:10.1021/jacs.6b03780

Sunzini, F., De Stefano, S., Chimenti, M. S., and Melino, S. (2020). Hydrogen Sulfide as Potential Regulatory Gasotransmitter in Arthritic Diseases. Int. J. Mol. Sci. 21 (4), 1180. doi:10.3390/ijms 21041180

Thorbjarnardottir, T., Olafsdottir, E. J., Valdimarsdottir, U. A., Olafsson, O., and Tryggvadottir, L. (2014). Oral Contraceptives, Hormone Replacement Therapy and Breast Cancer Risk: a Cohort Study of 16928 Women 48 Years and Older. Acta Oncologica 53 (6), 752-758. doi:10.3109/0284186x.2013.878471

Wang, R. (2010). Hydrogen Sulfide: the Third Gasotransmitter in Biology and Medicine. Antioxid. Redox Signaling 12 (9), 1061-1064. doi:10.1089/ars.2009.2938

Wang, R. (2012). Physiological Implications of Hydrogen Sulfide: a Whiff Exploration that Blossomed. Physiol. Rev. 92 (2), 791-896. doi:10.1152/ physrev.00017.2011

Wang, Y., Nishida, S., Boudignon, B. M., Burghardt, A., Elalieh, H. Z., Hamilton, M. M., et al. (2007). IGF-I Receptor Is Required for the Anabolic Actions of
Parathyroid Hormone on Bone. J. Bone Miner Res. 22 (9), 1329-1337. doi:10.1359/jbmr.070517

Whiteman, M., Li, L., Rose, P., Tan, C.-H., Parkinson, D. B., and Moore, P. K. (2010). The Effect of Hydrogen Sulfide Donors on Lipopolysaccharide-Induced Formation of Inflammatory Mediators in Macrophages. Antioxid. Redox Signaling 12 (10), 1147-1154. doi: $10.1089 /$ ars.2009.2899

Williams, F. M., Skinner, J., Spector, T. D., Cassidy, A., Clark, I. M., Davidson, R. M., et al. (2010). Dietary Garlic and Hip Osteoarthritis: Evidence of a Protective Effect and Putative Mechanism of Action. BMC Musculoskelet. Disord. 11, 280. doi:10.1186/1471-2474-11-280

Xu, Z.-S., Wang, X.-Y., Xiao, D.-M., Hu, L.-F., Lu, M., Wu, Z.-Y., et al. (2011). Hydrogen Sulfide Protects MC3T3-E1 Osteoblastic Cells against H2O2Induced Oxidative Damage-Implications for the Treatment of Osteoporosis. Free Radic. Biol. Med. 50 (10), 1314-1323. doi:10.1016/ j.freeradbiomed.2011.02.016

Xu, Z. S., Dai, F., Chen, J., Lv, M., Cheng, J. W., Zhang, X. M., et al. (2018). Experimental Research into the Potential Therapeutic Effect of GYY4137 on Ovariectomy-Induced Osteoporosis. Cell Mol Biol Lett 23, 47. doi:10.1186/ s11658-018-0114-0

Yan, X., Wu, H., Wu, Z., Hua, F., Liang, D., Sun, H., et al. (2017). The New Synthetic H2S-Releasing SDSS Protects MC3T3-E1 Osteoblasts against H2O2Induced Apoptosis by Suppressing Oxidative Stress, Inhibiting MAPKs, and Activating the PI3K/Akt Pathway. Front. Pharmacol. 8, 07. doi:10.3389/ fphar.2017.00007

Yang, J., Tang, R., Yi, J., Chen, Y., Li, X., Yu, T., et al. (2019). Diallyl Disulfide Alleviates Inflammatory Osteolysis by Suppressing Osteoclastogenesis via NFкB-NFATc1 Signal Pathway. FASEB j. 33 (6), 7261-7273. doi:10.1096/ fj.201802172r

Yang, M., Zhang, K., Zhang, X., Zhang, Z., Yin, X., He, G., et al. (2019). Treatment with Hydrogen Sulfide Donor Attenuates Bone Loss Induced by Modeled Microgravity. Can. J. Physiol. Pharmacol. 97 (7), 655-660. doi:10.1139/cjpp2018-0521

Yang, X., Hao, D., Zhang, H., Liu, B., Yang, M., and He, B. (2017). Treatment with Hydrogen Sulfide Attenuates Sublesional Skeletal Deterioration Following Motor Complete Spinal Cord Injury in Rats. Osteoporos. Int. 28 (2), 687-695. doi:10.1007/s00198-016-3756-7

Yu, F., Li, P., Song, P., Wang, B., Zhao, J., and Han, K. (2012). An ICT-Based Strategy to a Colorimetric and Ratiometric Fluorescence Probe for Hydrogen Sulfide in Living Cells. Chem. Commun. 48 (23), 2852-2854. doi:10.1039/ c2cc17658k

Zhao, Y., Pacheco, A., and Xian, M. (2015). Medicinal Chemistry: Insights into the Development of Novel H2S Donors. Handb Exp. Pharmacol. 230, 365-388. doi:10.1007/978-3-319-18144-8_18

Zhao, Y., Wang, H., and Xian, M. (2011). Cysteine-activated Hydrogen Sulfide (H2S) Donors. J. Am. Chem. Soc. 133 (1), 15-17. doi:10.1021/ ja 1085723

Zhou, Y., Wu, Z., Cao, X., Ding, L., Wen, Z., and Bian, J. S. (2016). HNO Suppresses LPS-Induced Inflammation in BV-2 Microglial Cells via Inhibition of NF-Kb and P38 MAPK Pathways. Pharmacol. Res. 111, 885-895. doi:10.1016/ j.phrs.2016.08.007

Conflict of Interest: The authors declare that the research was conducted in the absence of any commercial or financial relationships that could be construed as a potential conflict of interest.

Copyright ( $\odot 2021$ Hao, Wang, Fang, Bian, Gao and Li. This is an open-access article distributed under the terms of the Creative Commons Attribution License (CC BY). The use, distribution or reproduction in other forums is permitted, provided the original author(s) and the copyright owner(s) are credited and that the original publication in this journal is cited, in accordance with accepted academic practice. No use, distribution or reproduction is permitted which does not comply with these terms. 